\title{
Comparison of survival outcomes according of patients with metastatic gastric cancer receiving trastuzumab with systemic chemotherapy
}

\author{
Gi-Young Ha', Sung-Hyun Yang ${ }^{2}$, Hye-Jin Kang ${ }^{2}$, Hyo-Lak Lee' ${ }^{2}$ Jin Kim³ ${ }^{3}$ Yun-Ju Kim³ ${ }^{3}$ Hang-Jong Yu', Jong-Inn Lee', Sung-Ho Jin ${ }^{1}$ \\ Departments of ${ }^{1}$ Surgery, ${ }^{2}$ Hemato-Oncology, and ${ }^{3}$ Gastroenterology, Korea Cancer Center Hospital, Korea Institute of Radiological and Medical \\ Sciences, Seoul, Korea
}

Purpose: Currently, trastuzumab plus chemotherapy is the standard first-line therapy for human epidermal growth factor receptor 2 (HER2)-positive advanced or metastatic gastric cancer ( $\mathrm{mGC}$ ) or esophagogastric junction cancer. However, it is not clear whether the prognosis of HER2-positive mGC treated with trastuzumab plus chemotherapy is better than that of HER2-negative mGC treated with chemotherapy as the first-line therapy.

Methods: We performed a retrospective study comparing the prognosis of $\mathrm{mGC}$ according to first-line treatment with trastuzumab plus chemotherapy or chemotherapy only, at the Korea Cancer Center Hospital from 2011 to 2018. The Kaplan-Meier method and Cox proportional hazards model were used for univariate and multivariate survival analyses.

Results: The median overall survival of trastuzumab group was 26.1 months and that of chemotherapy group was 14.8 months $(P=0.047)$. Trastuzumab group had a longer median progression-free survival than chemotherapy group (23.4 vs. 9.2 months, $\mathrm{P}=0.026$ ). By univariate analysis, sex, age, World Health Organization (WHO) histology, HER2 status, primary tumor site, extent of disease, number of lesions, number of metastatic, measurability of disease, prior gastrectomy, and chemotherapy group are statistically significant. Using multivariate analysis, number of lesions, number of metastatic, prior gastrectomy, and trastuzumab group (hazard ratio, 0.594; 95\% confidence interval, 0.384$0.921 ; P=0.020$ ) were found to be independent prognostic factors of overall survival.

Conclusion: The result suggests prognosis of HER2-positive mGC treated by trastuzumab plus chemotherapy could be better than that of HER2-negative mGC treated by chemotherapy only. Well-designed prospective cohort studies are needed to confirm the results of this study. HER2 testing should be performed routinely in all patients newly diagnosed with $\mathrm{mGC}$.

Keywords: Stomach, Esophagogastric junction, Drug therapy, Trastuzumab, Survival

\section{INTRODUCTION}

Gastric cancer is the sixth most common cancer worldwide and the third leading cause of cancer-related death [1]. In 2015, gastric

Received: Sep 9, 2020 Revised: Nov 11, 2020 Accepted: Nov 18, 2020

Correspondence to: Sung-Ho Jin

Department of Surgery, Korean Cancer Center Hospital, 75 Nowon-ro, Nowon-gu, Seoul 01812, Korea

Tel: +82-2-970-2114, Fax:+82-2-978-2005

E-mail: peacejin0602@gmail.com

ORCID: Gi-Young Ha (https://orcid.org/0000-0002-5667-4122), Sung-Ho Jin (https://orcid.org/0000-0002-7459-5962)

Copyright (C) 2020 Korean Society of Surgical Oncology

This is an Open Access article distributed under the terms of the Creative Commons Attribution Non-Commercial License (http://creativecommons.org/licenses/by-nc/4.0) which permits unrestricted non-commercial use, distribution, and reproduction in any medium, provided the original work is properly cited. cancer accounted for approximately 783,000 deaths, as estimated by the World Health Organization (WHO) [2]. Most gastric cancer-related deaths are caused by metastasis or recurrence to the peritoneal surface, liver, and distant lymph nodes [3]. Systemic chemotherapy prolongs the survival of patients with recurrent, advanced, or metastatic gastric cancer (mGC). Palliative chemotherapy provides a better quality of life than the best supportive care alone for patients with mGC [3]. Therefore, systemic chemotherapy is generally accepted as the standard treatment of $\mathrm{mGC}$.

Currently, several chemical agents, such as platinum, fluoropyrimidine, taxane, and irinotecan, are available to treat gastric cancer. Although randomized studies have not clearly shown which combination regimen is the most efficient for treating $\mathrm{mGC}$, the platinum plus fluoropyrimidine regimen is generally recommended as a first-line therapy for this condition [4]. Comprehensive molecular characterization of gastric cancer has been studied ex- 
tensively. Therefore, target therapy to the human epidermal growth factor receptor 2 (HER2)-positive advanced gastric cancer has been established as the first-line therapy based on the Phase III Trastuzumab for Gastric Cancer (ToGA) trial $[5,6]$. Trastuzumab in combination with capecitabine or fluorouracil plus cisplatin has been the standard first-line therapy for patients with HER2-positive advanced or $\mathrm{mGC}$ or esophagogastric junction (EGJ) cancer $[7,8]$.

HER2 overexpression, determined by immunohistochemistry using a monoclonal antibody (HercepTest) and/or gene amplification by in situ hybridization, has been detected in $13 \%-22 \%$ of gastric or EGJ cancers; moreover, HER2-positive frequency is higher in EGJ cancers than in gastric cancers and in intestinal type than in diffuse type, according to the Lauren classification [9-11]. Although the clinical implications and prognostic effects of HER2 in breast cancer have been well documented, these are unclear in gastric and EGJ cancers [10-15]. HER2 positivity was not an independent prognostic factor among patients with mGCs and EGJ cancers who received first-line chemotherapy without trastuzum$\mathrm{ab}$ [16]. The ToGA trial showed that trastuzumab plus chemotherapy prolonged overall survival (OS) among patients with HER2-positive gastric and EGJ cancers. However, it is not clear whether the survival of patients with HER2-positive gastric and EGJ cancers treated with trastuzumab-based first-line chemotherapy is better than that of patients with HER2-negative gastric and EGJ cancers treated with other first-line chemotherapy regimens $[17,18]$.

This retrospective study aimed to compare the prognosis of HER2-positive metastatic, recurrent, or advanced gastric/EGJ cancers treated with trastuzumab with that of HER2-negative metastatic, recurrent, or advanced gastric/EGJ cancers treated with other chemotherapies.

\section{METHODS}

\section{Patient and data collection}

We recruited 1,086 patients with gastric cancer from July 2011 to June 2018. In total, 532 patients received palliative chemotherapy. Of these, 10 were treated mainly for other cancers (one of liver and nine of lung cancers). Of the patients that received palliative chemotherapy, 522 were hospitalized for gastric cancer. Among these gastric cancer patients, 89 were excluded from the study because they received radiotherapy, while four patients were excluded as they did not meet the inclusion criteria. Thus, 429 patients were selected for our study. These patients underwent palliative chemotherapy for mGC in the Department of Surgery, Department of Hematooncology, and Department of Gastroenterology of Korea Cancer Center Hospital, Seoul, Korea. We selected patients with
mGC who underwent palliative chemotherapy regardless of success following the 8th edition American Joint Committee on Cancer guidelines. Finally, 381 patients received only chemotherapy and 48 received chemotherapy and trastuzumab (Herceptin) therapy.

We collected and retrospectively analyzed patient clinical data including age (patients were grouped as older and younger than 65 years), sex, presence of metastasis or tumor recurrence, use of trastuzumab, primary tumor location (circumferential or longitudinal), metastatic location, WHO classification, WHO grade, Bormann type, underwent or did not undergo tumor reduction operation, and lymph node invasion and differentiation. We compared patients treated who received trastuzumab or other therapeutic regimens for clinical, progression-free survival (PFS), and OS data. Furthermore, the analysis was extended to compare patients with HER2-positive, HER2-negative, or HER2-unknown mGC.

The study was approved by the Institutional Review Board of Korea Cancer Center Hospital (IRB No. 2018-08-005) and performed in accordance with the principles of the Declaration of Helsinki. Written informed consent was waived.

\section{Statistical analysis}

Data were analyzed using IBM SPSS Statistics Korean version 23 (IBM Corp., Armonk, NY, USA), Kaplan-Meier, Cox regression analysis, and chi-square test. Result with a P-value $\leq 0.05$ was considered statistically significant.

\section{RESULTS}

This study was conducted at the Korea Cancer Center Hospital from July 2011 to June 2018. Table 1 presents the baseline characteristics of patients. Of the total, 48 patients received trastuzumab plus capecitabine/cisplatin (XP), and 381 received other chemotherapy regimens. The median patient age in the two groups at the index date was 65.5 and 64.0 years, respectively. Over two-thirds of the patients were male. Few variables differed significantly between the two groups: patients in the trastuzumab plus XP group were more cohesive $(\mathrm{P}<0.001)$, had HER2-positive cancers $(\mathrm{P}<0.001)$, and had a smaller number of lesions $(\mathrm{P}=0.046)$ than those in the other-regimen group. The primary tumor site, extent of disease, number of metastatic sites, measurability of disease, prior gastrectomy, and chemotherapy response were not significantly different between the two groups. The OS and PFS were significantly higher in the trastuzumab plus XP group than in the other group (26.1 and 14.8 months $[\mathrm{P}=0.047]$ vs. 23.4 and 9.2 months $[\mathrm{P}=0.011]$ ).

On univariate survival analyses, sex, age, WHO histology, HER2 status, primary tumor site, extent of disease, number of lesions, 
Table 1. Demographics and baseline characteristics of patients

\begin{tabular}{|c|c|c|c|}
\hline \multirow{2}{*}{ Characteristics } & \multicolumn{2}{|c|}{ First-line chemotherapy } & \multirow{2}{*}{ P-value } \\
\hline & Trastuzumab plus XP $(n=48)$ & Other regimens $(n=381)$ & \\
\hline Sex & & & 0.685 \\
\hline Male & $35(72.9)$ & $267(70.1)$ & \\
\hline Female & $13(27.1)$ & $114(29.9)$ & \\
\hline Median age (yr) & $65.5(42-89)$ & 64.0 (21-99) & 0.606 \\
\hline Age & & & 0.443 \\
\hline$\leq 64 \mathrm{yr}$ & $22(45.8)$ & $197(51.7)$ & \\
\hline$>64 \mathrm{yr}$ & $26(54.2)$ & $184(48.3)$ & \\
\hline WHO histology & & & $<0.001$ \\
\hline Cohesive & $33(68.8)$ & $134(35.2)$ & \\
\hline Poorly cohesive & 15 (31.3) & $247(64.8)$ & \\
\hline HER2 expression & & & $<0.001$ \\
\hline HER2-negative & 0 & $259(68.0)$ & \\
\hline HER2-positive & $48(100)$ & $11(2.9)$ & \\
\hline Unknown & 0 & $111(29.1)$ & \\
\hline Primary tumor site & & & 0.436 \\
\hline Esophagogastric junction & $8(16.7)$ & $82(21.5)$ & \\
\hline Gastric & 40 (83.3) & $299(78.5)$ & \\
\hline Extent of disease & & & 0.206 \\
\hline Metastatic & $41(85.4)$ & $295(77.4)$ & \\
\hline Recurrent & $7(14.6)$ & $86(22.6)$ & \\
\hline No. of lesions & & & 0.046 \\
\hline$\leq 2$ & $32(66.7)$ & $185(48.6)$ & \\
\hline$>2$ & $16(33.3)$ & $196(51.4)$ & \\
\hline No. of metastatic sites & & & 0.486 \\
\hline$\leq 2$ & 38 (79.2) & $317(83.2)$ & \\
\hline$>2$ & $10(20.8)$ & 64 (16.8) & \\
\hline Measurability of disease & & & 0.274 \\
\hline Measurable & $36(75.0)$ & $256(67.2)$ & \\
\hline Non-measurable & $12(25.0)$ & $125(32.8)$ & \\
\hline Prior gastrectomy & & & 0.311 \\
\hline No & $25(52.1)$ & $169(44.4)$ & \\
\hline Yes & $23(47.9)$ & $212(55.6)$ & \\
\hline Chemotherapy response & & & 0.188 \\
\hline Responder & 35 (72.9) & $241(63.3)$ & \\
\hline Non-responder & $13(27.1)$ & $140(36.7)$ & \\
\hline Median overall survival (mo) & 26.1 & 14.8 & 0.047 \\
\hline Median progression-free survival (mo) & 23.4 & 9.2 & 0.011 \\
\hline
\end{tabular}

Value are presented as number (\%) or median (range).

WHO, World Health Organization; HER2, human epidermal growth factor receptor 2; XP, capecitabine/cisplatin.

number of metastatic sites, measurability of disease, prior gastrectomy, and chemotherapy regimen are significantly associated with OS (Table 2). According to the multivariate survival analyses, trastuzumab group remained as a significant prognostic factor and showed remarkably better survival than other-regimen group (hazard ratio, 0.594; 95\% confidence interval, 0.384-0.921; $\mathrm{P}=$
0.020). And patients with small number of lesions, small metastatic sites, and history of prior gastrectomy also showed significantly better prognosis (Table 2).

According to Kaplan-Meier curves, the median OS of patients when trastuzumab was included as the first-line agent with chemotherapy (26.1 months) was significantly different $(\mathrm{P}=0.047)$ 
Table 2. Univariate and multivariate survival analyses

\begin{tabular}{|c|c|c|c|c|}
\hline \multirow{2}{*}{ Factor } & \multirow{2}{*}{$\begin{array}{c}\text { Univariate analysis } \\
\text { P-value }\end{array}$} & \multicolumn{3}{|c|}{ Multivariate analysis } \\
\hline & & HR & $95 \% \mathrm{Cl}$ & P-value \\
\hline Sex, male (vs. female) & 0.304 & & & \\
\hline $\mathrm{Age}_{1} \leq 64 \mathrm{yr}(\mathrm{vs} .>64 \mathrm{yr})$ & 0.156 & & & \\
\hline WHO criteria-based histology, cohesive (vs. poorly cohesive) & 0.060 & & & \\
\hline HER2 status, HER2-negative (vs. HER2-positive) & 0.157 & & & \\
\hline Primary tumor site, esophagogastric junction (vs. gastric) & 0.522 & & & \\
\hline Extent of disease, metastatic (vs. recurrent) & 0.666 & & & \\
\hline No. of lesions, $\leq 2$ (vs. $>2$ ) & 0.004 & 0.683 & $(0.496-0.939)$ & 0.019 \\
\hline No. of metastatic sites, $\leq 2$ (vs. $>2$ ) & 0.028 & 0.514 & $(0.371-0.711)$ & $<0.001$ \\
\hline Measurability of disease, measurable (vs. non-measurable) & 0.232 & & & \\
\hline Prior gastrectomy, no (vs. yes) & $<0.001$ & 2.928 & $(2.213-3.873)$ & $<0.001$ \\
\hline Chemotherapy, trastuzumab ${ }^{a)}$ (vs. other regimens ${ }^{\text {b) }}$ ) & 0.011 & 0.594 & $(0.384-0.921)$ & 0.020 \\
\hline
\end{tabular}

$\mathrm{HR}$, hazard ratio; $\mathrm{Cl}$, confidence interval; WHO, World Health Organization; HER2, human epidermal growth factor receptor 2; XP, capecitabine/cisplatin. a) Trastuzumab plus XP. ${ }^{b / I}$ ndicates FP, Fluorouracil/Ci spla tin; XP, mEOC, ECX, and mFOLFOX.
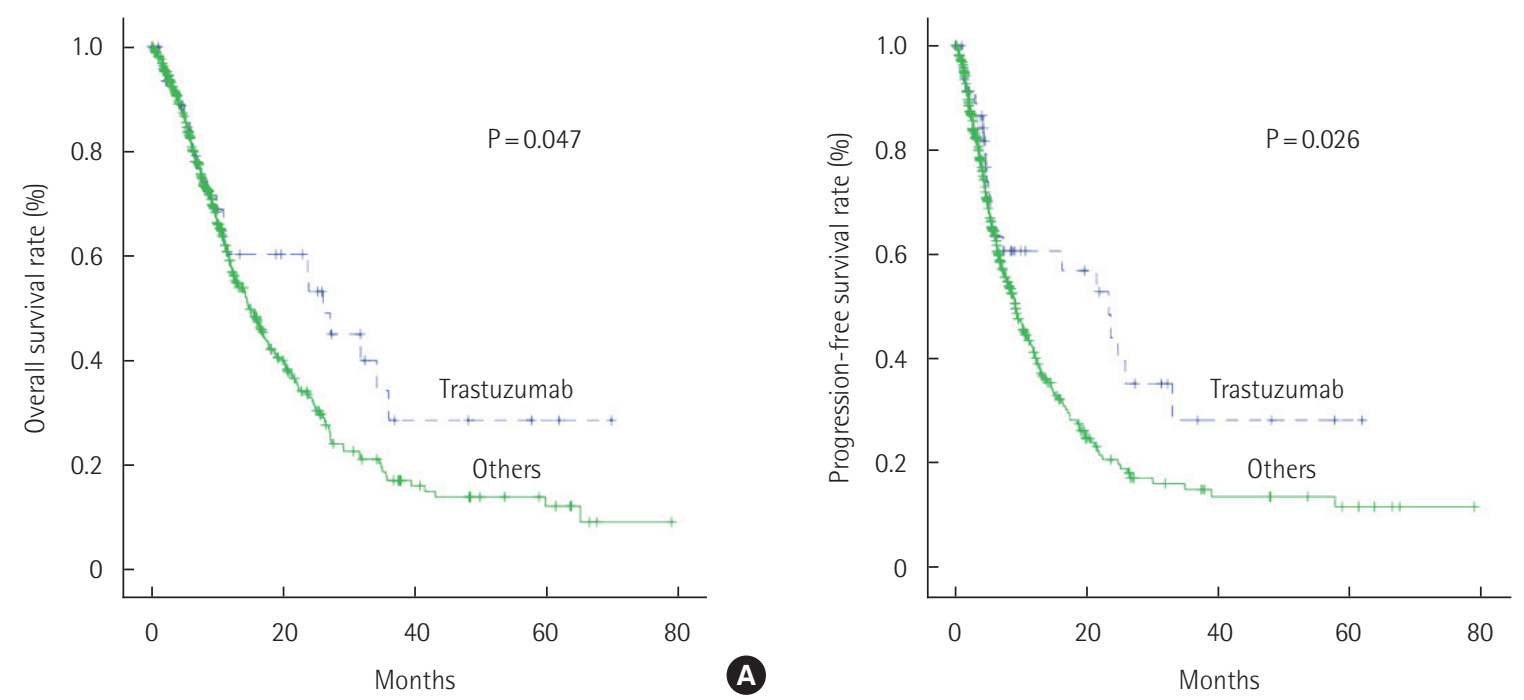

Fig. 1. Overall survival and progression-free survival rates among patients following trastuzumab-based and other therapeutic regimens. (A) Median overall survival time of patients who received trastuzumab was 26.1 months and of those who did not receive trastuzumab was 14.8 months. (B) Patients who received trastuzumab had a longer median progression-free survival time than those who did not receive trastuzumab (23.4 months vs. 9.2 months).

from that of patients who received only chemotherapy ( 14.8 months). Similarly, the median PFS of patients receiving trastuzumab plus chemotherapy (23.4 months) was significantly different from that of patients receiving only chemotherapy (9.2 months, $\mathrm{P}=0.026$ ) (Fig. 1).

A more detailed analysis was conducted by classifying the patients into HER2-positive $(\mathrm{n}=59)$, HER2-negative $(\mathrm{n}=259)$, and HER2-unknown groups $(\mathrm{n}=111)$. According to Kaplan-Meier curves, the median OS was 23.9 months in the HER2-positive group, 16.1 months in the HER2-negative group, and 14.6 months in the HER2-unknown group ( $\mathrm{P}=0.151)$. The median PFS was
21.6 months in the HER2-positive group, 10.3 months in the HER2-negative group, and 8.9 months in the HER2-unknown group $(\mathrm{P}=0.086)$ (Fig. 2).

Considering the high $\mathrm{P}$-values obtained in these analyses $(\mathrm{P}=$ 0.151 and $\mathrm{P}=0.086$ ), we classified to combine the HER-negative and HER2-unknown groups and repeated the analysis with only two groups: HER2-positive $(\mathrm{n}=59)$ and HER2-negative/unknown groups $(\mathrm{n}=370)$. According to Kaplan-Meier curves, the median OS was not significantly different between the HER2-positive (23.9 months) and HER2-negative/unknown groups (14.7 months, $\mathrm{P}=0.052$ ). In contrast, a significant difference was noted regarding 

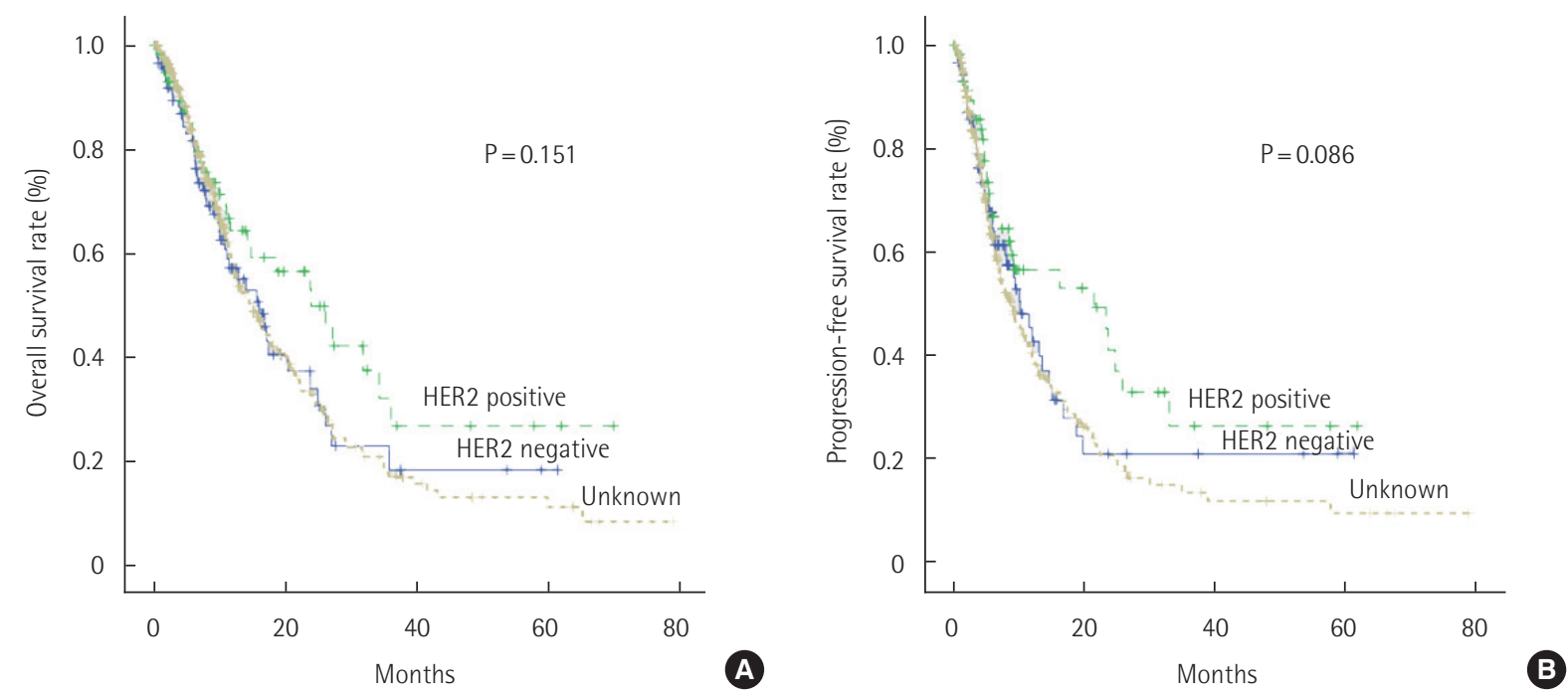

Fig. 2. Overall survival and progression-free survival rates among patients in the human epidermal growth factor receptor 2 (HER2)-positive, HER2-negative, and HER2-unknown groups. (A) Median overall survival time in the HER2-positive group was 23.9 months, HER2-negative group was 16.1 months, and HER2-unknown group was 14.6 months. (B) Median progression-free survival time in the HER2-positive group was 21.6 months, HER2-negative group was 10.3 months, and HER2-unknown group was 8.9 months.
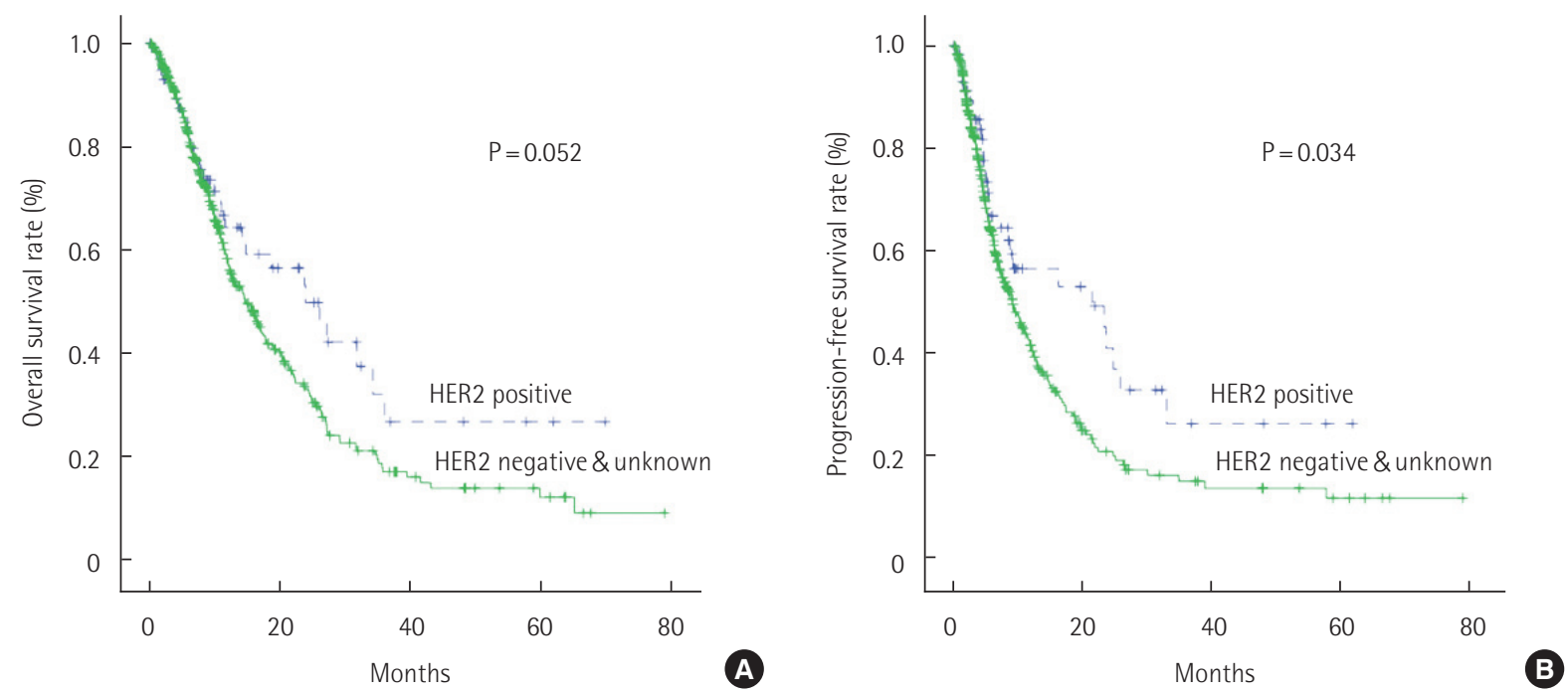

Fig. 3. Overall survival and progression-free survival rates among patients in the human epidermal growth factor receptor 2 (HER2)-positive (trastuzumab $n=48$, other regimens $n=11$ ), HER2-negative, and HER2-unknown groups. (A) Median overall survival time in the HER2-positive group was 23.9 months and the HER2-negative/unknown groups was 14.7 months. (B) Median progression-free survival time in the HER2-positive group was 21.6 months and the HER2-negative/unknown group was 9.2 months.

the median PFS between the HER2-positive (21.6 months) and HER2-negative/unknown groups (9.2 months, $\mathrm{P}=0.034$ ) (Fig. 3).

OS and PFS rates between patients with HER-positive gastric cancer with trastuzumab and those treated with other therapeutics. Median OS time of the patients who received trastuzumab was 26.1 months and of those who did not receive trastuzumab was 14.8 months $(\mathrm{P}=0.047)$. Patients who received trastuzumab had longer median PFS time than those who did not receive it
(23.4 months vs. 9.2 months, $\mathrm{P}=0.026$ ) (Fig. 4).

\section{DISCUSSION}

HER2 is a membrane-bound HER involved in the transmission of key signals controlling cell growth and survival. It is expressed in many epithelial tissues such as the breast, gastrointestinal tract, urinary tract, and heart. HER2 protein overexpression or HER2 gene 

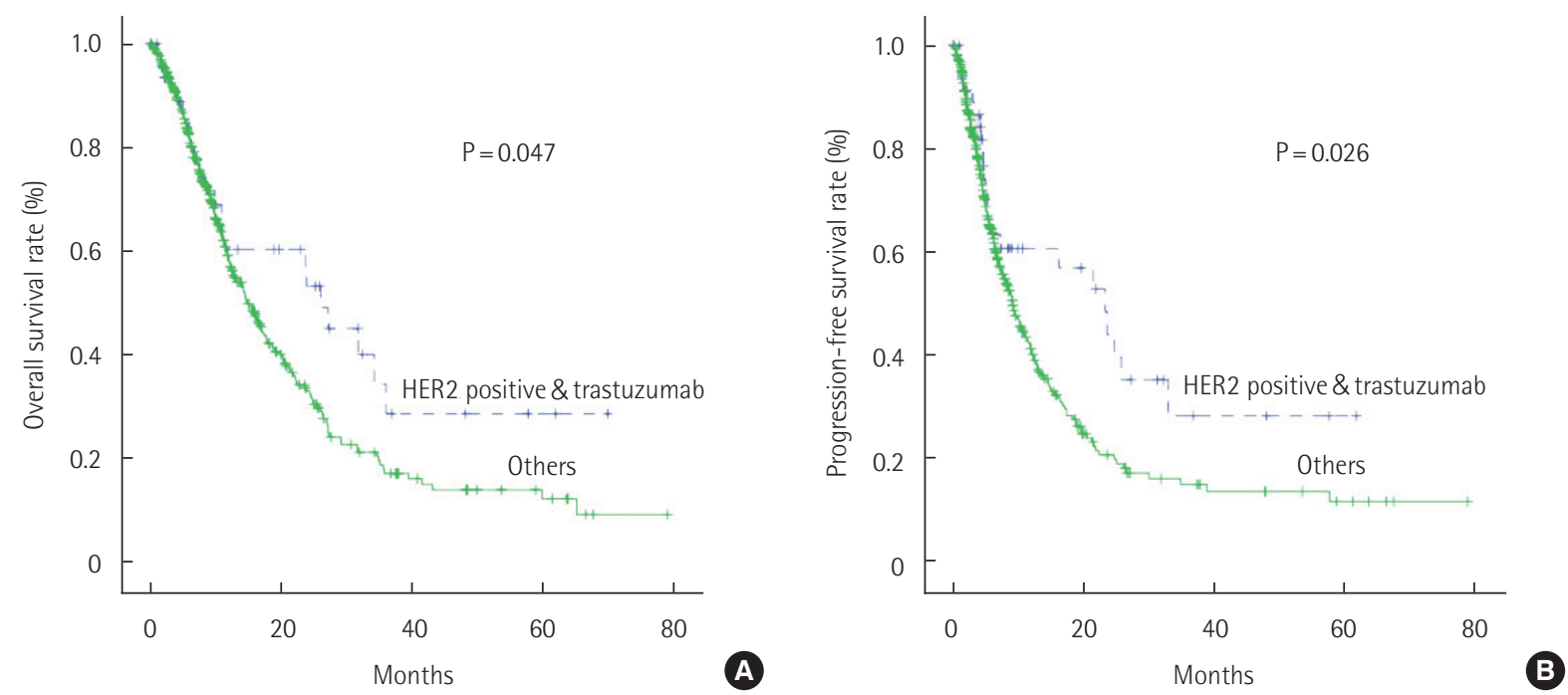

Fig. 4. Overall survival and progression-free survival rates between patients with human epidermal growth factor receptor 2 (HER2)-positive gastric cancer with trastuzumab and those treated with other therapeutics. (A) Median overall survival time of the patients who received trastuzumab was 26.1 months and of those who did not receive trastuzumab was 14.8 months. (B) Patients who received trastuzumab had longer median progression-free survival time than those who did not receive it (23.4 months vs. 9.2 months).

amplification plays a key role in transformation and tumorigenesis that leads to the development of breast, colon, bladder, ovary, endometrium, lung, uterine cervix, head and neck, esophagus, and gastric carcinomas [19-21].

HER2 overexpression and amplification have been detected in $10 \%-34 \%$ of invasive breast cancers; HER2-positive breast cancer correlates with poor prognosis and is a predictive factor of poor response to chemotherapy and endocrine therapies [6]. HER2 overexpression and gene amplification have been reported in 13\%$22 \%$ of gastric and EGJ cancers. HER2 positivity is noted at a higher incidence in EGJ cancers than in gastric cancers and similarly in the intestinal type than in the diffuse type [9-11]. Few studies reported that HER2 positivity was associated with a significantly worse prognosis in gastric and EGJ cancers [9,10,22-24], whereas other studies suggested no significant association between HER2 positivity and prognosis $[15,25,26]$. Therefore, the clinical implication and prognosis based on HER2 status in gastric and EGJ cancers remain unclear [10,12-15].

HER2 is a member of the HER family that includes HER1, HER3, and HER4. It has no natural ligand and lacks an extracellular ligand-binding domain; however, ligand binding to HER1, HER3, or HER4 leads to rapid receptor dimerization with HER2, which is a strongly preferred dimerization partner. HER2 heterodimers such as HER1-HER2, HER2-HER3, and HER2-HER4 strongly trigger the phosphorylation of intracellular tyrosine kinase domain and activate subsequent intracellular signal cascade, including Ras/RAF/MEK/ERK cascades. In addition, the HER2-
HER3 heterodimer has a more profound effect than either the HER2 or HER3 homodimer because it can also strongly activate the PI3K-Akt pathway [27]. In addition, in HER-2 positive cancers, HER2 spontaneously forms homodimers in cellular membranes $[1,15]$. Based on these unique features of HER2 in the EGFR (epidermal growth factor receptor) pathway, HER2-targeted therapy has been extensively studied and established as one of the standard treatments for breast and gastric cancers.

Trastuzumab is a humanized chimeric IgG1 monoclonal antibody that directly targets the extracellular domain of the HER2 protein. The key therapeutic mechanisms of trastuzumab are suggested to be the inactivation of intracellular HER2 signal cascade pathways, including Ras/MAPK and PI3K/Akt, leading to cell cycle arrest, reduction in angiogenesis, inhibition of extracellular-domain cleavage, and antibody-dependent cell-mediated cytotoxicity [28-30]. Trastuzumab has been shown to be effective against both metastatic HER2-positive breast and gastric cancers [17,18].

The ToGA trial was a prospective, phase 3 trial that demonstrated the prolongation of OS with trastuzumab plus chemotherapy $[17,18]$. The following data compare the general characteristics of the trastuzumab plus chemotherapy group in the ToGA trial with those of the trastuzumab plus chemotherapy group in our single-center study: number of patients, 294 vs. 48; age, 59.4 years vs. 65.5 years; proportion of men, $77 \%$ vs. $73 \%$; chemotherapy regimen, $87 \%$ vs. $100 \%$ for capecitabine and cisplatin, and 13\% vs. $0 \%$ for fluorouracil and cisplatin; primary tumor site, stomach: $90 \%$ vs. $83 \%$, gastroesophageal junction: $20 \%$ vs. $17 \%$; and patients with 
metastatic disease at study entry, 97 vs. $85 \%$. The survival data from the ToGA trial and our study were as follows: median OS, 13.8 vs. 26.1 months; PFS, 6.7 months vs. 23.4 months; and overall response rate, $47 \%$ vs. $72.9 \%$. These findings showed remarkable differences between patients in the groups receiving trastuzumab plus chemotherapy; however, the median OS (11.1 months vs. 14.8 months) and PFS (5.5 months vs. 9.2 months) showed considerable differences between the patient groups receiving chemotherapy only in the two studies. The good survival results of this study might be mainly attributed to the high proportion of ISH-positive/ IHC 3 + patients in this study ( $58 \%$ vs. ToGA trial $45 \%$ ) and the recent advances in the chemotherapy and target therapy of metastatic gastric and EGJ cancer.

Before trastuzumab became available, HER2 positivity itself was not a significant prognostic factor of patients with metastatic gastric and EGJ cancer [16]. Even now, it is still not clear whether the survival of patients with HER2-positive gastric and EGJ cancers treated with trastuzumab-based first-line chemotherapy is better than that of patients with HER2-negative gastric and EGJ cancers treated with other chemotherapies $[17,18]$. The results of this study suggest that the survival of patients with HER2-positive gastric and EGJ cancers could be better than that of patients with HER2-negative gastric and EGJ cancers when the former receive trastuzumab-based chemotherapy.

We acknowledge that there are many limitations and biases in this study. Furthermore, well-designed prospective cohort studies are needed to confirm our study results. More importantly, HER2 testing should be routinely performed in all patients newly diagnosed with advanced or mGCs or EGJ cancers, and trastuzumab-based chemotherapy should be actively administered to patients with HER2-positive gastric and EGJ cancers.

\section{CONFLICT OF INTEREST}

No potential conflict of interest relevant to this article was reported.

\section{REFERENCES}

1. Ferlay J, Soerjomataram I, Dikshit R, Eser S, Mathers C, Rebelo M, et al. Cancer incidence and mortality worldwide: sources, methods and major patterns in GLOBOCAN 2012. Int J Cancer 2015;136: E359-86.

2. Jung KW, Won YJ, Kong HJ, Lee ES; Community of Population-Based Regional Cancer Registries. Cancer statistics in Korea: incidence, mortality, survival, and prevalence in 2015. Cancer Res Treat 2018;50:303-16.
3. Wagner AD, Grothe W, Haerting I, Kleber G, Grothey A, Fleig WE. Chemotherapy in advanced gastric cancer: a systematic review and meta-analysis based on aggregate data. J Clin Oncol 2006;24:29039.

4. National Comprehensive Cancer Network (NCCN). NCCN Clinical Practice Guidelines in Oncology (NCCN Guidelines 2018) [Internet]. Plymouth Meeting (PA): NCCN; c2019 [cited 2020 Dec 29]. Available from: https://www.nccn.org/professionals/physician_gls/default.aspx.

5. Cancer Genome Atlas Research Network. Comprehensive molecular characterization of gastric adenocarcinoma. Nature 2014; 513:202-9.

6. Bang YJ, Van Cutsem E, Feyereislova A, Chung HC, Shen L, Sawaki A, et al. Trastuzumab in combination with chemotherapy versus chemotherapy alone for treatment of HER2-positive advanced gastric or gastro-oesophageal junction cancer (ToGA): a phase 3 , open-label, randomized controlled trial. Lancet 2010;376:687-97.

7. Ajani JA, D’Amico TA, Almhanna K, Bentrem DJ, Chao J, Das P, et al. Gastric cancer, version 3.2016, NCCN clinical practice guidelines in oncology. J Natl Compr Canc Netw 2016;14:1286-312.

8. Guideline Committee of the Korean Gastric Cancer Association (KGCA), Development Working Group \& Review Panel. Korean practice guideline for gastric cancer 2018: an evidence-based, multi-disciplinary approach. J Gastric Cancer 2019;19:1-48.

9. Tanner M, Hollmen M, Junttila TT, Kapanen AI, Tommola S, Soini Y, et al. Amplification of HER-2 in gastric carcinoma: association with Topoisomerase Ilalpha gene amplification, intestinal type, poor prognosis and sensitivity to trastuzumab. Ann Oncol 2005; 16:273-8.

10. Park DI, Yun JW, Park JH, Oh SJ, Kim HJ, Cho YK, et al. HER-2/ neu amplification is an independent prognostic factor in gastric cancer. Dig Dis Sci 2006;51:1371-9.

11. Lordick F, Bang YJ, Kang YK, Otero Reyes D, Manikhas GM, Shen L, et al. HER2-positive advanced gastric cancer: similar HER2-positivity levels to breast cancer. EJC Suppl 2007;5:272.

12. Begnami MD, Fukuda E, Fregnani JH, Nonogaki S, Montagnini AL, da Costa WL Jr, et al. Prognostic implications of altered human epidermal growth factor receptors (HERs) in gastric carcinomas: HER2 and HER 3 are predictors of poor outcome. J Clin Oncol 2011;29:3030-6.

13. Kim KC, Koh YW, Chang HM, Kim TH, Yook JH, Kim BS, et al. Evaluation of HER2 protein expression in gastric carcinomas: comparative analysis of 1,414 cases of whole-tissue sections and 595 cases of tissue microarrays. Ann Surg Oncol 2011;18:2833-40.

14. Nakajima M, Sawada H, Yamada Y, Watanabe A, Tatsumi M, Yamashita J, et al. The prognostic significance of amplification and overexpression of c-met and c-erb B-2 in human gastric carcino- 
mas. Cancer 1999;85:1894-902.

15. Gomez-Martin C, Garralda E, Echarri MJ, Ballesteros A, Arcediano A, Rodriguez-Peralto JL, et al. HER2/neu testing for antiHER2-based therapies in patients with unresectable and/or metastatic gastric cancer. J Clin Pathol 2012;65:751-7.

16. Allgayer H, Babic R, Gruetzner KU, Tarabichi A, Schildberg FW, Heiss MM. c-erbB-2 is of independent prognostic relevance in gastric cancer and is associated with the expression of tumor-associated protease systems. J Clin Oncol 2000;18:2201-9.

17 Qiu MZ, Li Q, Wang ZQ, Liu TS, Liu Q, Wei XL, et al. HER2-positive patients receiving trastuzumab treatment have a comparable prognosis with HER2-negative advanced gastric cancer patients: a prospective cohort observation. Int J Cancer 2014;134:2468-77.

18. Shitara K, Yatabe Y, Matsuo K, Sugano M, Kondo C, Takahari D, et al. Prognosis of patients with advanced gastric cancer by HER2 status and trastuzumab treatment. Gastric Cancer 2013;16:261-7.

19. Neve RM, Lane HA, Hynes NE. The role of overexpressed HER2 in transformation. Ann Oncol 2001;12 Suppl 1:S9-13.

20. Menard S, Pupa SM, Campiglio M, Tagliabue E. Biologic and therapeutic role of HER2 in cancer. Oncogene 2003;22:6570-8.

21. Rubin I, Yarden Y. The basic biology of HER2. Ann Oncol 2001;12 Suppl 1:S3-8.

22. Dang HZ, Yu Y, Jiao SC. Prognosis of HER2 over-expressing gastric cancer patients with liver metastasis. World J Gastroenterol 2012;18:2402-7.

23. Jorgensen JT, Hersom M. HER2 as a prognostic marker in gastric cancer: a systematic analysis of data from the literature. J Cancer.
2012;3:137-44.

24. Kim JW, Im SA, Kim M, Cha Y, Lee KH, Keam B, et al. The prognostic significance of HER2 positivity for advanced gastric cancer patients undergoing first-line modified FOLFOX-6 regimen. Anticancer Res 2012;32:1547-53.

25. Janjigian YY, Werner D, Pauligk C, Steinmetz K, Kelsen DP, Jager E, et al. Prognosis of metastatic gastric and gastroesophageal junction cancer by HER2 status: a European and USA International collaborative analysis. Ann Oncol 2012;23:2656-62.

26. Terashima M, Ochiai A, Kitada K, Ichikawa W, Kurahashi I, Sakuramoto $S$, et al. Impact of human epidermal growth factor receptor (EGFR) and ERBB2 (HER2) expressions on survival in patients with stage II/III gastric cancer, enrolled in the ACTS-GC study. J Clin Oncol 2011;29(15 Suppl):4013.

27. Nami B, Maadi H, Wang Z. Mechanisms underlying the action and synergism of trastuzumab and pertuzumab in targeting HER2-positive breast cancer. Cancers (Basel) 2018;10:342.

28. Pohlmann PR, Mayer IA, Mernaugh R. Resistance to trastuzumab in breast cancer. Clin Cancer Res 2009;15:7479-91.

29. Arnould L, Gelly M, Penault-Llorca F, Benoit L, Bonnetain F, Migeon C, et al. Trastuzumab-based treatment of HER2-positive breast cancer: an antibody-dependent cellular cytotoxicity mechanism? Br J Cancer 2006;94:259-67.

30. Mimura K, Kono K, Hanawa M, Kanzaki M, Nakao A, Ooi A, et al. Trastuzumab-mediated antibody-dependent cellular cytotoxicity against esophageal squamous cell carcinoma. Clin Cancer Res 2005;11:4898-904. 